SNUTP 97-146

\title{
Chiral symmetry breaking by a non-Abelian external field in $(2+1)$-dimensions
}

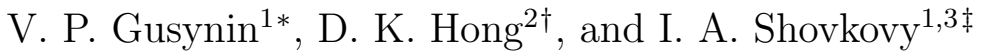 \\ ${ }^{1}$ Bogolyubov Institute for Theoretical Physics \\ 252143 Kiev, Ukraine \\ ${ }^{2}$ Department of Physics, Pusan National University \\ Pusan 609-735, Korea \\ ${ }^{3}$ Physics Department, University of Cincinnati \\ $\mathrm{OH}$ 45221-0011, USA
}

\begin{abstract}
We investigate the effect of a constant external non-Abelian field on chiral symmetry breaking in a $(2+1)$-dimensional Nambu-Jona-Lasinio model and in 3D QCD by solving the gap equation and the Bethe-Salpeter equation, and also by RG analysis. In the (2+1)-dimensional NJL model chiral symmetry breaking occurs for any weak coupling constant but in 3D QCD catalysis of chiral symmetry breaking does not occur.
\end{abstract}

11.30.Rd, 11.10.Kk, 11.10.Gh, 12.20.Ds

\footnotetext{
*Email address: vgusynin@gluk.apc.org

$\dagger$ Email address: dkhong@hyowon.cc.pusan.ac.kr

‡Email address: igor@physics.uc.edu
} 
Though there are increasing evidences for chiral symmetry breaking $(\chi \mathrm{SB})$ in QCD [1], we still do not understand clearly how it occurs, due to a lack of comprehensive understanding the QCD vacuum. One of the popular analytic methods to study $\chi \mathrm{SB}$ in QCD is by solving the Schwinger-Dyson (SD) equation for the Green functions, from whose solution one can extract the structure of the vacuum [2]. Most attention in SD analysis has been focused so far on the effect of the strong coupling in QCD; if the coupling is bigger than a critical coupling, the SD equation admits stable $\chi \mathrm{SB}$ solutions. But, the effect of self-interacting gluons in SD analysis has not been seriously considered except in the running coupling, even though it has been conjectured that the monopole condensate in QCD leads to color confinement and thus $\chi \mathrm{SB}$ [3], which is now shown rigorously in $N=2$ supersymmetric QCD [四].

Since the quark condensate under an external gluon field $A_{\mu}^{a}$ is related to the density of eigenvalue of Dirac operator $i \not D(A)$ at zero eigenvalue [5],

$$
\langle 0|\bar{q} q| 0\rangle^{A}=-\pi \rho_{A}(0),
$$

it is conceivable that a certain gluon field configuration which gives a constant eigenvalue density at small eigenvalue like a free $(0+1)$-dimensional spectrum is a source of $\chi \mathrm{SB}$. On the other hand, it has been recently found that an external magnetic field catalyzes chiral symmetry breaking in the Nambu-Jona-Lasinio (NJL) model [6] and in quantum electrodynamics [7 9]. The fact that a constant magnetic field enhances fermion dynamical masses was known from the papers [10]. Studying $(2+1)$-dimensional NJL model with discrete chiral symmetry, it was shown [11] also that chiral symmetry turns out spontaneously broken for any $B \neq 0$. However, what was shown in [6] is not just that the magnetic field enhances dynamical fermion masses created by supercritical NJL interaction, but that it catalyzes generating the mass even at the weakest attractive interaction and this effect is universal, i.e., model independent in $3+1$ and $2+1$ dimensions $s$. The physical reason for the magnetic catalysis of $\chi \mathrm{SB}$ is that, in the presence of external magnetic field $\vec{B}=B \hat{z}$, dimensional reduction occurs in fermion motion. The fermion spectrum becomes one-dimensional, $E= \pm\left|p_{z}\right|$, at energy much lower than the Landau gap $E \ll \sqrt{|e B|}$ and, for one-dimensional fermions, an arbitrarily weak attraction at scale $\mu=\sqrt{|e B|}$ leads to a strong attraction in the infrared region [9]. It is important that in [6, 7] continuous chiral symmetry was considered, that is far nontrivial problem since the appearance of Nambu-Goldstone bosons along with reduction to $D=0+1$ or $D=1+1$ dimensions might wash out symmetry breaking, as happens, for example, in the chiral $(1+1)$-dimensional Gross-Neveu model [13]. However, it was shown that notwithstanding dimensional reduction Goldstone boson propagators keep their three or four dimensional form.

In studying $\chi \mathrm{SB}$ in $\mathrm{QCD}$, it is natural to look for a gluon field that mimics a magnetic field and gives a $(1+1)$-dimensional spectrum for the Dirac operator. We consider this in a $(2+1)$-dimensional NJL model and in a $(2+1)$-dimensional $S U(2)$ gauge theory for simplicity. These theories in $2+1$ dimensions have been studied intensively recently, since they can serve not only as theoretical laboratories for investigating aspects of chiral symmetry breaking in

\footnotetext{
$\S$ For recent application of this effect in condensed matter physics see 12
} 
QCD but also describe the high temperature thermodynamics of 4-dimensional theories as well as certain planar condensed matter systems.

A constant and uniform chromomagnetic field for a non-Abelian gauge theory can be described by two different types of gauge potentials [14,15], a feature not available for the abelian case. As is known these two different potentials lead to two different energy spectra for fermions in such a field [14,16]. The non-Abelian external field which mimics a magnetic field and has the largest global symmetry including explicit translational invariance, is described by a constant gauge potential

$$
A_{\mu}=\frac{1}{2} A_{\mu}^{a} \tau^{a}, \quad \text { with } A_{1}^{1}=A_{2}^{2}=\sqrt{H / g}, \text { and others }=0
$$

where $\tau^{a}$ 's are the Pauli matrices $(a=1,2,3), g$ is the gauge coupling constant, and $H$ is a constant. Then, the field strength tensor,

$$
F_{\mu \nu}^{a} \frac{\tau^{a}}{2}=\frac{1}{i g}\left[D_{\mu}, D_{\nu}\right]
$$

so that $F_{12}^{3}=-F_{21}^{3}=-H$ and others are zero, where the covariant derivative $D_{\mu}=$ $\partial_{\mu}+i g A_{\mu}^{a} \tau^{a} / 2$. Under the external field, Eq. (2)), the fermion propagator satisfies

$$
\left[\gamma^{\mu}\left(i \partial_{\mu}-g A_{\mu}\right)-m\right] S(x-y)=i \delta(x-y),
$$

where $m$ is a fermion mass and we take the four-component reducible $(2+1)$-dimensional spinor for fermions. To find the fermion propagator we look for a solution of the form

$$
S(x-y)=i\left[\gamma^{\mu}\left(i \partial_{\mu}-g A_{\mu}\right)+m\right] \Delta(x-y),
$$

where the Fourier transform of $\Delta(x-y)$ satisfies the following equation:

$$
\left[P_{\mu} P^{\mu}-\frac{g}{4} \sigma^{\mu \nu} F_{\mu \nu}^{a} \tau^{a}-m^{2}\right] \Delta(p)=1
$$

where

$$
P_{\mu}=p_{\mu}-g A_{\mu}, \quad \sigma_{\mu \nu}=\frac{i}{2}\left[\gamma^{\mu}, \gamma^{\nu}\right], \quad\left\{\gamma^{\mu}, \gamma^{\nu}\right\}=2 g^{\mu \nu}
$$

With our choice of the vector potential (2) the equation (6) simplifies to

$$
\left[p^{2}-2 h(\vec{p} \cdot \vec{\tau})-2 h^{2}+2 h^{2} \tau^{3} \sigma^{12}-m^{2}\right] \Delta(p)=1,
$$

where we introduced the notation $h=\sqrt{g H} / 2$. The last equation can easily be solved and we find:

$$
\Delta(p)=\frac{1}{Q_{1} Q_{2}}\left[p^{2}-2 h^{2}+2 h \vec{p} \cdot \vec{\tau}-2 h^{2} \tau^{3} \sigma^{12}-m^{2}\right]
$$

where

$$
Q_{1,2}(p)=p_{0}^{2}-m^{2}-\left[\sqrt{\vec{p}^{2}+h^{2}} \pm h\right]^{2}
$$


Finally, the Fourier transform of the fermion propagator reads

$$
S(p)=i\left[\gamma^{\mu} \hat{P}_{\mu}+m\right] \Delta(p) .
$$

We see that if $m=0$ the pole of the propagator occurs at

$$
p_{0}= \pm\left(\sqrt{\vec{p}^{2}+h^{2}} \pm h\right) .
$$

At low momenta, $|\vec{p}| \ll h$, the spectrum changes drastically, it has a non-relativistic branch: $p_{0}= \pm \vec{p}^{2} /(2 h)$. It occurs that just this mode is responsible for chiral symmetry breaking in NJL model.

Now, we consider the (2+1)-dimensional NJL model with $N$ four-component fermions in the presence of the above non-Abelian field, described by the Lagrangian density

$$
\mathcal{L}=\bar{\psi} i \gamma^{\mu} D_{\mu} \psi+\frac{G}{2 N}\left[(\bar{\psi} \psi)^{2}+\left(\bar{\psi} i \gamma_{5} \psi\right)^{2}+\left(\bar{\psi} \gamma_{3} \psi\right)^{2}\right]
$$

which has $U(2 N)$ symmetry. In terms of auxiliary fields $\sigma, \tau$ and $\pi$ it can be rewritten as

$$
\mathcal{L}^{\prime}=\bar{\psi} i \gamma^{\mu} D_{\mu} \psi-\bar{\psi}\left(\sigma+\gamma^{3} \tau+i \gamma^{5} \pi\right) \psi-\frac{N}{2 G}\left(\sigma^{2}+\pi^{2}+\tau^{2}\right) .
$$

In the large $N$ limit the vacuum is determined by the stationary point of the effective action, obtained by integrating over the fermions:

$$
e^{i S_{\mathrm{eff}}(\sigma, \tau, \pi)}=\int[d \psi d \bar{\psi}] e^{i \int d^{3} x \mathcal{L}^{\prime}},
$$

or

$$
S_{\mathrm{eff}}=-\frac{N}{2 G} \int d^{3} x\left(\sigma^{2}+\pi^{2}+\tau^{2}\right)-i \operatorname{Tr} \log \left[i \gamma^{\mu} D_{\mu}-\left(\sigma+i \gamma_{5} \pi+\gamma_{3} \tau\right)\right] .
$$

Since the vacuum should respect translational invariance, we need to calculate the effective action for constant auxiliary fields to find the vacuum. In this case, the effective action is just $S_{\text {eff }}(\sigma, \tau, \pi)=-V(\sigma, \tau, \pi) L^{2} T$, where $L^{2} T$ is the spacetime volume and $V$ is the effective potential. The potential $V$ depends only on the chiral invariant $\rho^{2}=\sigma^{2}+\pi^{2}+\tau^{2}$ and for its calculation it is sufficient to consider a configuration with $\tau=\pi=0$ and $\sigma=$ const. In the proper-time formalism we get for $V(\sigma)$ :

$$
V(\sigma)=\frac{N \sigma^{2}}{2 G}-\frac{i}{2} \int_{0}^{\infty} \frac{d s}{s} \int \frac{d^{3} p}{(2 \pi)^{3}} \operatorname{tr} e^{i s\left[P_{\mu} P^{\mu}-\frac{g}{4} \sigma^{\mu \nu} F_{\mu \nu}^{a} \tau^{a}-\sigma^{2}\right]} .
$$

The matrix in the exponent of (17) has two eigenvalues (compare with (10))

$$
\lambda_{1,2}=p_{0}^{2}-\sigma^{2}-\left[\sqrt{\vec{p}^{2}+h^{2}} \pm h\right]^{2},
$$

each of which has $4 N$ degeneracy. Thus we write 


$$
\begin{aligned}
V(\sigma) & =\frac{N \sigma^{2}}{2 G}+2 N \int_{1 / \Lambda^{2}}^{\infty} \frac{d s}{s} \int \frac{d^{3} p}{(2 \pi)^{3}}\left[e^{-s\left[p_{3}^{2}+\sigma^{2}+\left(\sqrt{\vec{p}^{2}+h^{2}}-h\right)^{2}\right]}\right. \\
& \left.+e^{-s\left[p_{3}^{2}+\sigma^{2}+\left(\sqrt{\vec{p}^{2}+h^{2}}+h\right)^{2}\right]}-2 e^{-s\left(p_{3}^{2}+\vec{p}^{2}\right)}\right]
\end{aligned}
$$

where we made a rotation to Euclidean space, subtracted the part corresponding to free fermions $(\sigma=0, h=0)$ and introduced the ultraviolet cutoff $\Lambda$. The effective potential up to the order of $\Lambda^{0}$ is computed to be

$V(\rho)=\frac{N \rho^{2}}{2 G}+\frac{N}{\pi}\left[-\frac{\rho^{2} \Lambda}{\sqrt{\pi}}+\frac{\rho^{3}+\left(\rho^{2}+4 h^{2}\right)^{3 / 2}}{3}-h^{2} \sqrt{\rho^{2}+4 h^{2}}-\frac{\rho^{2} h}{2} \ln \left(\frac{2 h+\sqrt{\rho^{2}+4 h^{2}}}{\rho}\right)\right]$,

where we substituted $\sigma$ by the chiral invariant $\rho$. The expression (20) agrees with that one derived in [17], except the $S U(3)$ color group was considered there. The stationary condition for the effective potential gives the gap equation:

$$
0=\frac{N \rho}{G}+\frac{N}{\pi}\left[-\frac{2 \rho \Lambda}{\sqrt{\pi}}+\rho^{2}+\rho\left(\rho^{2}+4 h^{2}\right)^{1 / 2}-\rho h \ln \left(\frac{2 h+\sqrt{\rho^{2}+4 h^{2}}}{\rho}\right)\right] .
$$

As $h \rightarrow 0$, we recover the known gap equation

$$
\rho^{2}=\rho \Lambda\left(\frac{1}{\sqrt{\pi}}-\frac{\pi}{2 \Lambda G}\right)
$$

It admits a nontrivial solution only if the coupling $G$ is supercritical, $G>G_{c}=\pi^{3 / 2} / 2 \Lambda$. The magnetic field changes the situation: at $h \neq 0$, it is easy to see that a nontrivial solution exists at all $G>0$. The reason for this is that the magnetic field enhances the interaction in the infrared region: at $h \neq 0$ the last term in (21) is responsible for the existence of a solution at any $G>0$. The nontrivial solution always minimizes the effective potential, if the four-Fermi coupling $G>0$, and therefore is the vacuum solution. Thus, unlike the NJL model with $h=0$, chiral symmetry breaking occurs for any value of $G>0$. In particular, when $G \rightarrow 0$,

$$
\bar{\rho}=4 h \exp \left(-\frac{\pi}{h G}\right)
$$

while $\bar{\rho} \simeq 0.4 h$ when $G \lesssim G_{c}$, the critical coupling for chiral symmetry breaking of the NJL model in the absence of the external field. Since $\bar{\rho}$ defines the fermion mass in the Lagrangian density (14), we find that the fermions get a dynamical mass, which is $m_{\text {dyn }}=$ $4 h \exp [-\pi /(h G)]$ for weak coupling. To analyze the supercritical region $G \gtrsim G_{c}$, it is useful to introduce the dynamical mass $m_{0}$, defined by the Eq.(22). For weak fields $\left(h \ll m_{0}\right)$, we find

$$
m_{\mathrm{dyn}}=\bar{\rho}=m_{0}\left(1+\frac{3 h^{2}}{4 m_{0}^{2}}\right)
$$

i.e. $m_{\text {dyn }}$ increases with $h$. 
If the cutoff $\Lambda=h$, the calculations get much simpler and the reason for the catalysis is easy to understand. Let us now introduce a dimensionless coupling $\lambda \equiv h G$. Then the effective potential becomes $(\rho \ll h)$

$$
V(\rho)=N h\left[\frac{2 h^{2}}{3 \pi}+\frac{\rho^{2}}{2 \lambda}+\frac{1}{4 \pi} \rho^{2}\left(\ln \frac{\rho^{2}}{16 h^{2}}-\frac{4}{\sqrt{\pi}}+3\right)\right]+O(1)
$$

from which we can read off the scaling behavior of the coupling $G$ by taking a different renormalization point as

$$
\left.\frac{1}{\lambda(\mu)} \equiv(N h)^{-1} \frac{d^{2} V}{d \rho^{2}}\right|_{\mu}=\frac{1}{\lambda}+\frac{1}{2 \pi}\left(\ln \frac{\mu^{2}}{16 h^{2}}-\frac{4}{\sqrt{\pi}}+6\right) .
$$

The dynamical mass of a fermion, which will be invariant under the change of the renormalization point, is of the order of

$$
m_{\text {dyn }} \simeq 4 h \exp \left(-\frac{\pi}{h G}+\pi+\frac{2}{\sqrt{\pi}}-3\right) .
$$

This result is very similar to magnetic catalysis, where an arbitrarily weak magnetic field catalyzes chiral symmetry breaking, though the motion of fermions at low energy is not dynamically reduced to one dimension by the uniform chromomagnetic field. The reason for catalysis of $\chi \mathrm{SB}$ by the uniform chromomagnetic field is that the spectrum of fermions changes at low energies, $E \ll h$, so that the scaling dimension of fermions is the same as that of fermions in $(1+1)$-dimensions and thus the four-Fermi interaction becomes a relevant operator. This can be easily seen by performing the renormalization group analysis similar to that which was applied to the ordinary magnetic catalysis [9].

Let us expand the fermion field as

$$
\Psi(\vec{r}, t)=\int_{k} \sum_{A} \psi_{A}(t, k) e^{i \vec{k} \vec{r}} u_{A}(\vec{k})
$$

where $u_{A}(\vec{k})$ are the eigenfunctions of the operator $\vec{\alpha} \cdot\left(\vec{k}-g \overrightarrow{A^{a}} \frac{\tau^{a}}{2}\right)$ with eigenvalues $E_{A}=$ $\alpha\left(\sqrt{\vec{k}^{2}+h^{2}}+\beta h\right)$ each of which is doubly degenerate. ( $A$ denotes collectively $\left.\alpha, \beta= \pm\right)$. After Fourier-transforming in $t$, we find that the kinetic term in the action is

$$
S_{0}=\sum_{A, i} \int \frac{d \omega}{2 \pi} \frac{d^{2} \vec{k}}{(2 \pi)^{2}} \tilde{\psi}_{A i}^{\dagger}(\omega, k)\left(\omega-E_{A}\right) \tilde{\psi}_{A i}(\omega), \quad i=1,2,
$$

where $\tilde{\psi}_{A i}(\omega, k)$ is the Fourier transform of $\psi_{A i}(t, k)$ and the spinors $u_{A i}$ are chosen to be normalized as $u_{A i}^{\dagger} u_{A j}=\delta_{A B} \delta_{i j}$.

When $|\vec{k}| \ll h$, the effect of the external field is important. It changes the energy spectrum drastically. To derive the low energy effective action, we integrate out the modes with $|\omega|,|\vec{k}|>h$. Then, the kinetic term in the effective action becomes

$$
S_{0}=\sum_{\alpha, i} \int_{|\omega|,|\vec{k}|<h} \frac{d \omega}{2 \pi} \frac{d^{2} \vec{k}}{(2 \pi)^{2}}\left[\tilde{\psi}_{\alpha i,-}^{\dagger}\left(\omega-\alpha \frac{\vec{k}^{2}}{2 h}\right) \tilde{\psi}_{\alpha i,-}+\tilde{\psi}_{\alpha i,+}^{\dagger}(\omega-2 \alpha h) \tilde{\psi}_{\alpha i,+}\right]+\cdots
$$


where we have expanded the energy eigenvalues $E_{A}$ in powers of momentum and the elipsis denotes the terms with higher powers of momenta.

To determine the scaling dimensions of the fermion modes, consider a transformation,

$$
\omega \rightarrow s \omega, \quad \vec{k} \rightarrow s^{1 / 2} \vec{k} \quad \text { with } \quad s<1 .
$$

Since the kinetic term must be invariant under the scaling transformation, we find $\tilde{\psi}_{\alpha i,-}$ has scaling dimension $-3 / 2$, while $\tilde{\psi}_{\alpha i,+}$ has -1 . Namely, in the coordinate space, $\psi_{\alpha i, \pm}(\vec{r}, t)$, the Fourier transform of $\tilde{\psi}_{\alpha i, \pm}$, has scaling dimension $1 / 2$ and 1 , respectively. We see that the component $\psi_{\alpha i,-}$ is more relevant in the infrared region than the component $\psi_{\alpha i,+}$. Because of the change in the energy spectrum due to the external field, the scaling dimension of fermion field changes for the component $\psi_{\alpha i,-}$ at low energy as if the spacetime is dimensionally reduced from $2+1$ to $1+1$. Because of this dimensional reduction, the four-Fermi interaction of $\psi_{\alpha i,-}$ is marginal. Now, let us further integrate out the modes of $s h<\omega<h, s^{1 / 2} h<$ $|\vec{k}|<h$ to find the change in the four-Fermi interaction. The one-loop correction to the four-Fermi coupling in the leading order in $1 / N$ is given then

$$
\delta G=\frac{-i G^{2}}{N} \int_{s h<\omega<h, s^{1 / 2} h<|\vec{k}|<h} \operatorname{Tr}[S(k) S(k)]=-\frac{G^{2} h}{\pi} \ln s .
$$

In terms of the dimensionless coupling $\lambda$, we find

$$
\beta(\lambda)=s \frac{\partial \lambda}{\partial s}=-\frac{1}{\pi} \lambda^{2} .
$$

Therefore, the four-Fermi interaction becomes a relevant operator at quantum level if $G>0$, which at low energy leads to strong attraction between fermion and antifermion to form a condensate. The dynamical fermion mass, the RG invariant scale, is $\left.m_{\mathrm{dyn}} \simeq h \exp [-\pi / h G)\right]$, which is, in leading order in the coupling expansion, the same as the dynamical mass we obtained by solving the gap equation (27) for weak coupling $\lambda$ at $\mu=h$, where the RG analysis is reliable.

The (2+1)-dimensional $S U(2)$ gauge theory is more complicated than the 3D NJL model, since not only the fermions but also the gauge field propagate nontrivially under the external uniform chromomagnetic field. The gauge field propagator (in Feynman gauge) in momentum space is determined as a solution to the equation:

$$
\left[\mathcal{P}_{\mu} \mathcal{P}^{\mu} g_{\alpha \beta}-2 i g F_{\alpha \beta}^{a} I^{a}\right] S^{\beta \gamma}(p)=-i \delta_{\alpha}^{\gamma}
$$

where $\mathcal{P}_{\mu}=p_{\mu}-g I^{a} A_{\mu}^{a}$ and $I^{a}$ are the generators in the adjoint representation. As is seen, to solve this equation one has to invert a matrix with double indices, Lorentz and internal. The structure in Lorentz indices, however, can be simplified considerably. That is due to the fact that the only nondiagonal Lorentz matrix appearing in the equation is $F_{\alpha \beta}^{3}$, and it commutes with $g_{\alpha \beta}$. So, we represent the gluon propagator through the projectors which diagonalize the $F_{\alpha \beta}^{3}$-matrix:

$$
S^{\beta \gamma}(p)=-i \sum_{j=-1}^{1} S^{j}(p) A_{j}^{\beta \gamma}
$$


where

$$
\begin{gathered}
A_{0}^{\beta \gamma}=g^{\beta \gamma}+\frac{F^{3 \beta \nu} F_{\nu}^{3} \gamma}{H^{2}}, \quad A_{ \pm 1}^{\beta \gamma}=\frac{1}{2}\left[\mp i \frac{F^{3 \beta \gamma}}{H}-\frac{F^{3 \beta \nu} F_{\nu}^{3} \gamma}{H^{2}}\right], \\
\sum_{j=-1}^{1} A_{j}^{\beta \gamma}=g^{\beta \gamma}, \quad F_{\nu}^{3 \beta} A_{j}^{\nu \gamma}=i H j A_{j}^{\beta \gamma} .
\end{gathered}
$$

Thus, we obtain the equation for $S^{j}$

$$
\left[p^{2}-4 h^{2}\left(\left(I^{1}\right)^{2}+\left(I^{2}\right)^{2}\right)-4 h(\vec{p} \cdot \vec{I})+8 j h^{2} I^{3}\right] S^{j}=1,
$$

which is easily solved by a simple inversion of $3 \times 3$ matrices. The result reads:

$$
S^{j}=\frac{a_{j}+b(\vec{p} \vec{I})+c\left(I^{3}\right)^{2}+j d I^{3}+f\left[(\vec{p} \vec{I})-2 h j I^{3}\right]^{2}}{E_{j}},
$$

where

$$
\begin{aligned}
& a_{j}=\left(p^{2}-4 h^{2}\right)^{2}-16 h^{2} \vec{p}^{2}-64 h^{4} j^{2}, \quad b=4 h\left(p^{2}-4 h^{2}\right), \\
& c=-4 h^{2}\left(p^{2}-4 h^{2}\right), \quad d=-8 h^{2}\left(p^{2}-8 h^{2}\right), \quad f=16 h^{2}, \\
& E_{j}=\left(p^{2}-8 h^{2}\right)\left(p^{2}-4 h^{2}\right)^{2}-16 h^{2} \vec{p}^{2}\left(p^{2}-4 h^{2}\right)-64 h^{4} j^{2}\left(p^{2}-8 h^{2}\right) .
\end{aligned}
$$

We find that under the external chromomagnetic field the gauge field is not massless, instead its propagator has poles at nonzero $p$, all of order $h$, satisfying $\left(p^{2}-8 h^{2}\right)\left(p^{2}-4 h^{2}\right)^{2}-$ $16 h^{2} \vec{p}^{2}\left(p^{2}-4 h^{2}\right)-64 h^{4} j^{2}\left(p^{2}-8 h^{2}\right)=0, \quad j=0, \pm 1$. Seven of these poles correspond to massive modes while the remaining two describe tachyonic modes signaling instability of the perturbative vacuum in the external chromomagnetic field (2) (analog of the Nielsen-Olesen instability in four-dimensional Yang-Mills theory for abelian configurations of chromomagnetic field [18] (* Since we are interested in infrared dynamics of fermions with energy and momenta $E,|\vec{p}| \ll h$, the presence of tachyonic modes of order $h$ is not very important. In fact we will be working in an effective low energy theory where $h$ serves as ultraviolet cutoff. Further we need the gauge propagator which is approximately constant for $E,|\vec{p}| \sim 0$,

$$
S_{\alpha \beta}^{a b}=\frac{i}{8 h^{2}} g_{\alpha \beta}\left(2 \delta^{a b}-\delta^{3 a} \delta^{3 b}\right)+\frac{i}{6 h^{2}} \frac{F_{\alpha \beta}}{H} \varepsilon^{3 a b}+\frac{i}{3 h^{2}} \frac{\left(F^{2}\right)_{\alpha \beta}}{H^{2}}\left(\delta^{a b}-\delta^{3 a} \delta^{3 b}\right) .
$$

Since the gauge field is massive, the gauge field exchange interaction will generate a fourFermi interaction at low energy $E \ll h$ :

$$
O_{4}(x)=-\frac{g^{2}}{48 h^{2}}\left[(\bar{\psi} \psi)^{2}+\left(\bar{\psi} i \gamma_{5} \psi\right)^{2}+\left(\bar{\psi} \gamma_{3} \psi\right)^{2}\right]+\cdots
$$

where the ellipsis denotes other than NJL interaction terms. For a while we assume that only NJL-like interactions play important role in the generation of a dynamical mass. Then, the

\footnotetext{
${ }^{* *}$ For a thorough investigation of the instability of non-Abelian fields given by constant potentials see [15].
} 
effective four-Fermi interaction generated by the massive gauge field is repulsive $\left(-\frac{g^{2}}{48 h^{2}}<0\right)$. Therefore, even though the four-Fermi interaction for the $\tilde{\psi}_{\alpha i,-}$ component of the fermion is marginal as we have shown in RG analysis of the 3D NJL model, its $\beta$-function is positive and it becomes an irrelevant operator at one-loop. Thus we may conclude that the chromomagnetic field does not induce chiral symmetry breaking in 3D QCD.

Since, while deriving the effective four-fermion interaction, we omitted terms other than those in (44) in order to apply the RG analysis, below we confirm our conclusion by analysis of the gap equation for a fermion dynamical mass. There are two equivalent approaches one can follow in studying mass generation, that of Schwinger-Dyson equations [8,9], and that of Bethe-Salpeter (BS) equation [7]. We consider here the BS equation for a gapless Nambu-Goldstone (NG) boson, composed of a fermion and antifermion, which must appear when the chiral symmetry is spontaneously broken.

The homogeneous BS equation for the NG bound state reads:

$$
\rho=-4 \pi \alpha \int \frac{d^{3} p}{(2 \pi)^{3}} \frac{\tau^{a}}{2} \gamma^{\mu} S(p) \rho S(p) \frac{\tau^{b}}{2} \gamma^{\nu} S_{\mu \nu}^{a b},
$$

where $\alpha=g^{2} /(4 \pi)$, and the Bethe-Salpeter wave function for the bound state is given in general as

$$
\rho=\gamma^{5}\left(A+\tau^{3} \sigma^{12} B+(\vec{\gamma} \cdot \vec{\tau}) C\right) .
$$

Since the dynamics responsible for fermion mass generation is due to momenta near $p=0$, we can neglect the momentum dependence in the gauge field propagator in the BS equation. Thus, the functions $A, B, C$ become momentum independent and Eq. (45) turns out to be an algebraic system of equations.

By direct substitution of (46) in the BS equation, we observe that the equation for coefficient $C$ decouples from the set of equations for $A$ and $B$. The latter is:

$$
\begin{aligned}
A & =\frac{i \pi \alpha}{24 h^{2}} \int \frac{d^{3} p}{(2 \pi)^{3}} \frac{\left[6 h^{2}+13\left(p^{2}-m^{2}\right)\right] A+\left[6 h^{2}-16\left(p^{2}+2 \vec{p}^{2}-m^{2}\right)\right] B}{Q_{1}(p) Q_{2}(p)}, \\
B & =\frac{i \pi \alpha}{24 h^{2}} \int \frac{d^{3} p}{(2 \pi)^{3}} \frac{\left[78 h^{2}-16\left(p^{2}-m^{2}\right)\right] A+\left[78 h^{2}-23\left(p^{2}+2 \vec{p}^{2}-m^{2}\right)\right] B}{Q_{1}(p) Q_{2}(p)} .
\end{aligned}
$$

In the limit $m \rightarrow 0$, this reduces to the following

$$
\begin{aligned}
& A \simeq-\frac{\alpha}{64 h} \ln \left(\frac{h}{m}\right)(A+B), \\
& B \simeq-\frac{13 \alpha}{64 h} \ln \left(\frac{h}{m}\right)(A+B),
\end{aligned}
$$

or in other words, we obtain

$$
1 \simeq-\frac{7 \alpha}{32 h} \ln \left(\frac{h}{m}\right)
$$

Thus, there is no solution satisfying the condition $m \ll h$, which agrees with the result of $\mathrm{RG}$ analysis (analysis of an equation for $C$-coefficient shows no nontrivial solution, too). 
While 3D QCD itself is known to be unstable in perturbation theory [19], and might lead to generating a mass for Yang-Mills fields nonperturbatively [20], the presence of an external chromomagnetic field enhances this instability. A chromomagnetic field influences the behavior of charged vector particles more strongly than that of fermions and further study is needed to make decisive conclusion about the magnetic catalysis in 3D QCD. Perhaps, simultaneous study of generating chiral condensate and a condensate of vector particles would shed light on this problem. The absence of chiral condensate in a chromomagnetic field in 3D QCD may cast doubts upon straightforward application of the results of a magnetic catalysis in QED4 [7-9] to QCD4 [21].

In conclusion, we investigated the effect of a constant external non-Abelian field on chiral symmetry breaking in a (2+1)-dimensional Nambu-Jona-Lasinio model and in 3D QCD. We studied the effect by gap equation analysis and RG analysis and found that in the $(2+1)$ dimensional NJL model chiral symmetry breaking occurs for any weak coupling constant (in accordance with earlier findings [17]) but catalysis of chiral symmetry breaking does not occur in 3D QCD.

\section{ACKNOWLEDGMENTS}

We are grateful to V.A. Miransky for helpful remarks and F. Hawes for correcting the manuscript. The final version of the paper was written when one of us (V.P.G.) was staying at the Special Research Centre for Subatomic Structure of Matter, the University of Adelaide, Australia. He would like to thank Prof. A. Thomas and Dr. A. Williams and all other members of the Centre for hospitality. This work is supported in part by Swiss National Science Foundation grant CEEC/NIS/96-98/7 IP 051219 (V.P.G.), by the academic research fund of Ministry of Education, Republic of Korea, Project No. BSRI-97-2413 (D.K.H.), by the KOSEF through SRC program of SNU-CTP (D.K.H.), by the U.S. Department of Energy grant \#DE-FG02-84ER40153 (I.A.Sh.), and by Foundation of Fundamental Researches of Ministry of Sciences of the Ukraine under grant N 2.5.1/003 (V.P.G., I.A.Sh.). One of the authors (D.K.H.) wishes to acknowledges the partial financial support of the Korea Research Foundation made in the program year of 1997. 


\section{REFERENCES}

[1] For recent review, see C. D. Roberts and A. G. Williams, Progr. Part. Nucl. Phys. 33, 477 (1994).

[2] V. A. Miransky, Dynamical Symmetry Breaking in Quantum Field Theories (World Scientific, 1993), and references therein.

[3] S. Mandelstam, Phys. Reports 23, 245 (1976); G. 't Hooft, in 1981 Cargése Summer School Lectures on Fundamental Interactions, NATO Adv. Study Inst. Series B: Phys., vol. 85, edited by M. Lévy et al. (Plenum Press, New York, 1982); Nucl. Phys. B190, 455 (1981).

[4] N. Seiberg and E. Witten, Nucl. Phys. B426, 19 (1994); B431, 484 (1994).

[5] T. Banks and A. Casher, Nucl. Phys. B169, 103 (1980); E. Marinari. G. Parisi, and C. Rebbi, Phys. Rev. Lett. 47, 1795 (1981); H. Leutwyler and A. Smilga, D46, 5607 (1992).

[6] V. P. Gusynin, V. A. Miransky, and I. A. Shovkovy, Phys. Rev. Lett. 73, 3499 (1994); Phys. Rev. D52, 4718 (1995); Phys.Lett. B349, 477 (1995).

[7] V. P. Gusynin, V. A. Miransky, and I. A. Shovkovy, Phys. Rev. D 52, 4747 (1995); Nucl. Phys. B 462, 249 (1996); V.P. Gusynin and I.A. Shovkovy, Phys. Rev. D 56, 5251 (1997).

V. P. Gusynin, "Magnetic catalysis of chiral symmetry breaking in QED at finite temperature", hep-ph/9709339.

[8] C. N. Leung, Y. J. Ng and A. W. Ackley, Phys. Rev. D 54, 4181 (1996); D.-S. Lee, C. N. Leung, Y. J. Ng, Phys. Rev. D 55, 6504 (1997).

[9] D. K. Hong, Y. Kim, and S. Sin, Phys. Rev. D 54, 7879 (1996); D. K. Hong, Seoul National University Report, SNUTP 97-053, hep-ph/9707432.

[10] S. Kawati, G. Konisi, and H. Miyata, Phys. Rev. D28, 1537 (1983); S.P. Klevansky and R.H. Lemmer, Phys. Rev., D39, 3478 (1989); I. V. Krive and S. A. Naftulin, Phys. Rev. D46, 2737 (1992).

[11] K. G. Klimenko, Z. Phys. C54, 323 (1992).

[12] K. Farakas and N. E. Mavromatos, "Gauge-theory approach to planar doped antiferromagnets and external magnetic fields", cond-mat/9710188.

[13] E. Witten, Nucl. Phys. B145, 110 (1978).

[14] L. S. Brown and W. I. Weisberger, Nucl. Phys. B157, 285 (1979).

[15] H. Leutwyler, Nucl. Phys. B179, 129 (1981).

[16] D. Ebert and V. Ch. Zhukovsky, "Chiral phase transitions in strong chromomagnetic fields at finite temperature and dimensional reduction", hep-ph/9701323.

[17] K. G. Klimenko, B. V. Magnitsky, and A. S. Vshivtsev, Nuovo Cim. A107, 439 (1994); Teor. Mat. Fiz. 101, 391 (1994).

[18] H. Nielsen and P. Olesen, Nucl. Phys. B144, 376 (1978).

[19] J. M. Cornwall, "On one-loop gap equations for the magnetic mass in $d=3$ gauge theory", hep-th/9710128.

[20] D. Karabali and V. P. Nair, Int. J. Mod. Phys. A12, 1161 (1997).

[21] I. A. Shushpanov and A. V. Smilga, Phys. Lett. B402, 351 (1997). 